\title{
ERNAUX OU LA TRAVERSÉE DES GENRES : ENTRE PASSION SIMPLE ET SE PERDRE
}

Joëlle Papillon, Université de Toronto

Journal du dehors et La vie extérieure, Les armoires vides et L'événement, Une femme et « Je ne suis pas sortie de ma nuit », Passion simple et Se perdre : I'œuvre d'Annie Ernaux semble vouloir se penser en paire. Dans le cas de ces deux dernières paires, la filiation des textes se fait encore plus serrée, au point presque de fusionner en un seul texte publié deux fois, d'abord sous la forme de récit (biographique, autobiographique ou autofictif), puis sous celle dite de « texte brut » du journal intime. Ce redoublement de publications - où, en apparence, le contenu est le même alors que la forme seule a changé - indique un questionnement des genres et une méfiance envers et le récit et le journal, tous deux jugés insuffisants pour représenter le réel de façon satisfaisante, surtout au niveau de la vérité de l'émotion si chère à Ernaux. Le moyen privilégié alors pour construire ce réel est I'addition des publications et des vérités, complémentaires et contradictoires. Dans son journal publié en 1997 «Je ne suis pas sortie de ma nuit », Ernaux se dit d'ailleurs convaincue « que I'unicité, la cohérence auxquelles aboutit une œuvre [...] doivent être mises en danger toutes les fois que c'est possible » (JSN : 12). Aux limites de la cohérence, effectivement, elle nous présente ce journal des plus intimes qu'est Se perdre; si le journal intime est le miroir de soi, c'est un soi tourmenté, torturé, que nous présente Ernaux, dévasté par un désir/amour/passion, marqué par la jalousie et la possessivité, transformé par le désintérêt puis la défection de l'amant, vécus de façon traumatique par la narratrice.

Passion simple s'écrit en 1991 tout de suite après la rupture et est publié la même année; Se perdre sort dix ans plus tard, en 2001, comme pour en célébrer l'anniversaire. Le trauma du réel a-t-il été apprivoisé entretemps ? Peut-être. Reste que Se perdre devient par la publication le texte second (pour nous, lecteurs) tout en restant (pour Ernaux) le texte premier, I'origine, la source, la preuve. Car le journal intime expose l'auteure d'une façon particulière, donnant à lire à tous la preuve de ses épreuves. Quelle importance donner à cette traversée entre les genres ? Comment justifier ce besoin du diaristique qui se fait sentir dans l'œuvre ernalienne à I'aube des années 2000 ? Tout se joue, pour Ernaux, dans la question de la vérité, qui n'est certainement pas perçue comme extérieure ou objective ici, mais qui se place résolument dans le registre de l'intime et de l'affectif. Dans la préface à Se perdre, Ernaux justifie ce geste de la publication par la découverte de cette vérité : « Je me suis aperçue qu'il y avait dans ces pages [Se perdre] une « vérité » autre que celle contenue dans Passion simple. Quelque chose de cru et de noir, sans salut, quelque chose de l'oblation » (SP: 15).

http://www.brocku.ca/cfra/voixplurielles06-01/index.html 


\section{Frontières génériques}

Si les trois premières publications d’Ernaux sont généralement considérées comme ses trois seuls romans, l'ensemble de ses livres subséquents décline la question générique à tous les temps : récit, autobiographie, autofiction, biographie, journal intime, journal croisé, journal du dehors, la liste s'allonge et les catégories possibles se forment et se déforment pour accommoder des textes hybrides et indisciplinés. Au fil des livres s'observe une méfiance grandissante envers le roman, qui protège l'auteure grâce au voile de la fiction. Dans Passion simple Ernaux attaque « la forme romanesque où les apparences sont sauves »(PS: 70); hors du romanesque, donc, l'écrivaine se compromet, elle met en danger sa réputation. La terreur engendrée par cette mise en danger de l'écriture et du soi est la preuve pour Ernaux de la valeur et de la pertinence du travail, tel que l'illustre d'ailleurs le mot de la fin de Se perdre : « Ce besoin que j'ai d'écrire quelque chose de dangereux pour moi, comme une porte de cave qui s'ouvre,

où il faut entrer coûte que coûte » (SP: 377). Sans frayeur, sans coût personnel, l'écriture demeure fausse, sans intérêt pour Ernaux. Le roman est donc ici hors de question.

\section{A. Le récit / I'autofiction}

En fait, Ernaux oppose dans Passion simple le romanesque de la passion vécue à la référentialité stricte du texte en train de s'écrire :

Tout ce temps, j'ai eu l'impression de vivre ma passion sur le mode romanesque, mais je ne sais pas, maintenant, sur quel mode je l'écris, si c'est celui du témoignage, voire de la confidence telle qu'elle se pratique dans les journaux féminins, celui du manifeste ou du procès-verbal, ou même du commentaire de texte (PS: 30-31).

Les interventions métatextuelles de ce genre abondent dans Passion simple, proposant des étiquettes génériques possibles pour le texte, mais sans jamais se fixer. Les étiquettes proposées convoquent des genres aux frontières du littéraire ou considérés comme mineurs, mais qui en revanche se revendiquent du vrai et de la confession : le témoignage, la confidence, le procès-verbal - ce qui souligne le désir de la narratrice d'inscrire son récit dans un pacte référentiel. Pourtant, elle retient suffisamment d'information quant à son identité de personne ou de personnage pour problématiser une lecture autobiographique, la narratrice demeurant anonyme dans le texte. Les biographèmes contenus au sein du texte et la signature par initiales «A. E. » présente en quatrième de couverture incitent tout de même le rapprochement entre Annie Ernaux sujet extra-textuel, A. E. sujet péritextuel, et la narratrice qui partage les mots de A. E. Le péritexte déclinant de trancher, une lecture de Passion simple en tant que récit autofictif semble autorisée, ou du moins n'est-elle pas découragée.

Mais le récit demeure problématique, comme le démontrent nombre de commentaires métatextuels, dont celui-ci, inséré dans une note en bas de page, où la narratrice commente le changement de temps verbal qui se produit à la fin du texte :

http://www.brocku.ca/cfra/voixplurielles06-01/index.html 
Je passe de l'imparfait, ce qui était - mais jusqu'à quand ? -, au présent - mais depuis quand ? - faute d'une meilleure solution. Car je ne peux rendre compte de l'exacte transformation de ma passion pour A., jour après jour, seulement m'arrêter sur des images, isoler des signes d'une réalité dont la date d'apparition - comme en histoire générale - n'est pas définissable avec certitude. (PS: 66-67)

Ici, Ernaux souligne ce que Passion simple fait (arrêts sur images; mise en relief de signes), tout en inscrivant en creux la place où Se perdre viendra s'installer dix ans plus tard afin de combler les manques (recherche des dates d'apparition - chacune des petites morts et des petites naissances; dégagement des certitudes historiques qui agissent comme preuves). Cette idée de recensement des signes de la passion obsède le texte, et s'y réinscrit sans cesse : « Je ne fais pas le récit d'une liaison, je ne raconte pas une histoire [...]. J'accumule seulement les signes d'une passion, [...] comme si cet inventaire allait me permettre d'atteindre la réalité de cette passion »(PS: 31$)$. La réalité chez Ernaux ne se laisse donc capturer que dans l'accumulation de signes disparates et éparpillés que l'auteure se fait un devoir de collectionner, d'identifier et d'exposer.

Cet assemblage hétéroclite, revendiqué dans Passion simple et évident dans Se perdre, est le signe d'une écriture hybride. Déjà le récit autofictif tend vers le journal (ou n'arrive que difficilement à s'en détacher) et on sent l'inscription du diaristique qui informe le récit. On le constate, par exemple, vers la fin du texte, où, après un long blanc, le texte reprend par «Maintenant c'est avril » (PS: 66), ou plus clairement encore quelques pages plus loin, dans la section finale du texte, qui est datée en retrait « février 91 »(PS: 71), à la manière du journal. C'est que le livre en train de s'écrire ne se laisse pas clore. Toujours la réalité confronte le texte, dont elle empêche l'achèvement, la mort en quelque sorte, et la narratrice tente de le garder avec elle le plus longtemps possible, elle hésite à le mettre au monde. Pour le retenir, elle l'allonge de considérations quotidiennes, le complète par le récit ponctuel d'événements postérieurs. Le récit, donc, appelle le journal, le désire et inscrit sa place à même son corps.

\section{B. Le journal intime}

À la valeur unificatrice du récit, le journal oppose le fragment, sous forme de notes, de miettes, qui relèvent à la fois de l'instantané et de l'interrompu. Le piège du journal ? La vision restreinte, c'est-à-dire son incapacité à considérer la situation avec une vue d'ensemble qui relierait les événements et créerait un sens. C'est que la mémoire ne peut pas organiser le texte de la même façon que dans les récits intimes écrits dans l'après-coup de l'événement. Autre différence majeure entre le journal et le récit: I'itérativité telle que discutée par Gérard Genette dans Figures III. Les récits sont en général itératifs en ce sens où la narratrice y raconte une fois ce qui s'est passé plusieurs fois (par exemple: « je lui remettais les lettres que je lui écrivais » (PS: 37), où I'imparfait souligne la répétition de l'acte). Les journaux, au contraire, sont généralement singulatifs c'est-à-dire que la narratrice y raconte une fois ce qui s'est passé une fois, et plusieurs fois ce qui s'est passé plusieurs fois. Il y a donc une esthétique de l'accumulation 
nécessaire (voire de l'énumération) dans le journal, qui en général fait défaut aux récits. Dans le journal, la répétition se lit, mais ne s'écrit pas.

Les théoriciens du journal intime (Michèle Leleu, Alain Girard et Béatrice Didier pour la tradition française) s'entendent pour définir le journal intime comme un texte composé de fragments datés, recueillis sur une base régulière par un seul auteur, et qui demeure généralement désorganisé. Notons que les journaux intimes publiés par Ernaux déjouent cette dernière caractéristique, par le fait que dans les deux cas (trois si nous comptons le texte hybride de L'événement) le journal se concentre sur un seul thème: la maladie de la mère dans « Je ne suis pas sortie de ma nuit », I'avortement dans L'événement, la liaison amoureuse dans Se perdre. Publier un journal intime relève à tout coup d'une pratique subversive, compte tenu de la transgression inévitable entre les sphères publique et privée. Le journal est en fait assez nouvellement reconnu comme littéraire, et son statut de genre est toujours contesté: on le traite comme une forme mineure au sein du paysage autobiographique, lui-même considéré comme mineur par des critiques qui mettent en doute sa littérarité par de vieilles mais toujours vigoureuses attaques quant à son prétendu narcissisme, comme le rappelle Philippe Vilain dans son Défense de Narcisse. Plusieurs critiques féministes de l'intime ont d'ailleurs proposé que cette dévaluation du journal était sans doute due en partie à la perception du journal intime comme activité codée féminine, et donc reléguée au statut de passe-temps.

Mais l'espoir du journal pour remonter la hiérarchie des textes intimes est sans conteste sa prétention à la vérité: puisque j'écris ce texte pour moi, en ne me supposant d'autre lectrice que moi, je n'ai aucune raison de me mentir ou d'embellir la vérité. Comme à la Cour, je jure de dire la vérité, toute la vérité et rien que la vérité. Ce gage de vérité est bien sûr présent de diverses façons dans tous les genres de l'intime, mais là où le journal puise sa supériorité morale est dans son écriture au ras de l'événement, au ras de l'émotion. Tous les récits intimes doivent en effet - malgré leurs promesses réitérées - se soumettre à la reconstruction de la vérité par la mémoire, et toute reconstruction implique d'abord une construction (donc une création, une invention) et ensuite l'appel à la mémoire, source de déplacements et de mensonges (volontaires ou non) s'il en est. Par son écriture quotidienne, le journal se placerait en quelque sorte - et en théorie - à l'abri de la mémoire et de ses égarements, plus proche du réel, dans ce qu'on a nommé diversement l'écriture vraie, l'écriture brute voire l'écriture urgente, ou encore l'écriture nécessaire.

\section{II. « Sauver la vie »}

Quant aux fonctions du journal intime pour Ernaux, elles sont également doubles. D’abord, il s'agit, comme elle le dit dans les premières pages de Se perdre de « sauver la vie » (SP: 13$)$, d'enregistrer la

http://www.brocku.ca/cfra/voixplurielles06-01/index.html 
passion, d'accumuler les preuves contre l'oubli. Ces preuves sont construites à différents niveaux, notamment dans la promesse d'une écriture vraie, c'est-à-dire non retravaillée.

Se perdre nous livre en effet - si I'on en croit la narratrice dans la préface - les entrées de son journal intime du 27 septembre 1988 au 9 avril 1990, complètes et intactes, avec pour seule réécriture le remplacement des noms de personnes par des initiales:

Je n'ai rien modifié ni retranché du texte initial en le saisissant sur ordinateur. Les mots qui se sont déposés sur le papier pour saisir des pensées, des sensations à un moment donné ont pour moi un caractère aussi irréversible que le temps : ils sont le temps luimême (SP: 15).

Que l'auteure tienne à préciser cette intégralité et cette intégrité du texte en dit long sur ce désir de coller au réel et d'être reçue, lue par le lecteur en tant que réel. Ernaux utilise d'ailleurs le même type de pacte de vérité diaristique dans la préface de « Je ne suis pas sortie de ma nuit »:

Je [livre ces pages ] telles qu'elles ont été écrites, dans la stupeur et le bouleversement que j'éprouvais alors. Je n'ai rien voulu modifier dans la transcription de ces moments où je me tenais près d'elle [de la mère], hors du temps (JSN: 13).

Notons en passant que ce renchérissement sur la véracité des propos, propos qui se répercutent dans les récits autofictifs qui font pendant aux journaux intimes, renforcent justement la lecture de ces textes en tant qu'autofictions, les journaux intimes fonctionnant alors comme preuves référentielles annexées au récit autofictif publié auparavant.

Le système de datation propre au journal fonctionne également comme preuve, en ce qu'il découpe le temps à l'aune du vécu, et qu'il dépose des bornes, lentement, pierre à pierre, qui à la relecture seulement prennent sens, deviennent des points de repère pour la reconstruction de I'histoire personnelle. Car la narratrice du journal précise bien qu'elle se relit sans cesse. Dans ces pages qui lui parlent d'elle, qui la raconte, elle cherche à comprendre son histoire et à décrypter les actions de l'amant afin, l'espèret-elle, de surprendre la preuve qu'elle est aimée de lui. Chaque geste, chaque parole, mais surtout chaque absence, chaque silence est scruté, interrogé et comparé à la série d'autres gestes et paroles, absences et silences qui se déploie le long des pages. Ainsi, la narratrice se base-t-elle sur son journal pour surveiller la fréquence des visites de l'amant:

Je compte les jours, attachant un signe de plus en plus négatif à leur écoulement sans appel téléphonique. Mais il a, lui, sans doute, une autre notion du temps, qu'il ne calcule même pas. Cette absence de calcul a un sens, cependant, le peu de nécessité que je dois avoir dans sa vie (SP: 196-197).

Elle spécifie textuellement qu'elle « note ces choses pour comparer avec ce qui précède et suivra » (SP: 227), ce qu'elle fait sur le mode: «Moments de lucidité. Il est évident que S. en a marre de moi. Inversement: le 12 mai, sa passion pour moi était évidente » (SP: 185). Elle procède donc à un inventaire 
ou encore à un relevé statistique de la présence ou de l'absence, de l'attention ou de l'indifférence de I'amant envers elle.

Puisque la narratrice affirme tenir un journal intime de façon plutôt continue depuis l'adolescence, elle ne fait pas que comparer l'amant à lui-même, mais elle le compare également aux hommes de son passé. Ce système de comparaisons élargit considérablement l'espace temporel du journal, ouvrant résolument le journal à la mémoire :

il vient le 4 août, répétition extraordinaire de 1963. Dans mon inconscient, il y a identité de quelque chose, à vingt-six ans de distance: force de l'attente, du désir. La relative jeunesse de S. entre aussi pour une part importante. C'est la permanence de I'homme dans son triomphe viril et doux, si doux, peau, cheveux, m'embrassant comme j'étais embrassée à dix-huit ans, dans le petit sentier près du cimetière, par D., à Yvetot, me désirant comme les garçons de Sées, et mieux que certains étudiants jadis, mieux sans doute que Ph. en Italie (SP: 227).

Le journal, chronique du présent, accueille ainsi en fait la totalité de l'expérience vécue de la diariste, qui voyage vers son passé grâce aux repères temporels que sont les dates. La narratrice signale son habitude de consulter ses vieux journaux intimes, ce qui lui permet de comparer ses états affectifs d'hier et d'aujourd'hui : « Les souvenirs de même nature [...] sont ainsi superposés et réduits à leurs dénominateurs communs. Le présent, inconnu, est ramené au passé, connu » (Gasparini 2007¹: 155). Les vieux journaux intimes font de nouveau figure de preuves, en ce que la narratrice prétend sa mémoire confirmée par le texte écrit par une autre elle-même. Le journal intime, du moins tel que pratiqué par Ernaux, n'échappe donc pas aux constructions mémorielles, et s'établit beaucoup plus sur un va-et-vient entre présent et passé, qui laisse voir un sujet fragile, marqué par l'incertitude et la crainte de l'abandon amoureux, s'appuyant sans cesse sur l'expérience passée afin de mieux évaluer la précarité de sa situation présente.

Pour que cette stratégie fonctionne et que la comparaison soit la plus efficace possible, il est nécessaire de se plier à l'exercice des détails, ce que la diariste fait méticuleusement, en recensant les gestes, les paroles, mais aussi, plus subtilement peut-être, les expressions et les pensées qui ont fait événement :

Ses paroles dans la cuisine, « ce sera dur», ensuite ses yeux, dans le fauteuil, avant son départ. La dernière fois, dans la chambre, sa douceur, ses mots érotiques, avec son accent russe, ses «tu es magnifique » murmurés. Écrire cela : je me suis aperçue que j'avais perdu une lentille. Je l'ai retrouvée sur son sexe. (Pensé : Zola perdait son lorgnon dans les seins des femmes. Moi je [perds] ma lentille sur le sexe de mon amant !) Je compare avec la nuit de Leningrad, comme il a changé, sa gaucherie alors. Je crois que ça s'appelle la passion maintenant (SP: 52-53).

Le geste de la diariste se comprend ainsi comme celui du collectionneur, qui recueille avec passion chacun des spécimens, en pressent tout de suite la valeur unique, et le dépose avec tendresse entre les pages

\footnotetext{
${ }^{1}$ Je remercie Valérie Dusaillant-Fernandes d'avoir porté à mon attention cet article de Philippe Gasparini.
} 
d'un livre. Chaque détail comme chaque spécimen n'a aucune valeur en lui-même, seul, et ce n'est qu'en tant que collection qu'il fait sens, qu'il devient lisible.

\section{Possession et contrôle}

Mais, outre la sauvegarde de la vie, le journal intime prend chez Ernaux une autre fonction, celle un peu moins candide de la possession de l'amant, et de la reprise de contrôle sur sa vie dont elle a l'impression qu'elle lui échappe. Le journal devient en effet un lieu de résistance à l'emprise de l'amant, un domaine privé où la hiérarchie s'inverse et où elle parvient à le dominer en l'emprisonnant par ses mots, en le transformant en objet textuel. À ce sujet, I'on peut penser aux remarques inquiètes de Philippe Vilain - dont on sait qu'il fut l'amant d'Ernaux - à propos de la toute-puissance de l'auteure, pour lui dangereuse et déloyale: « c'est ce désir pour un auteur de s'approprier et d'emprisonner l'autre par l'écriture, d'en faire en quelque sorte sa chose, le jouet de son pouvoir » (Vilain: 52). Ce commentaire de Vilain fait référence à la joute à laquelle se sont livrés Ernaux et Vilain par autofictions interposées, et que Barbara Havercroft a brillamment analysé ailleurs.

Dans le cas de Se perdre, le but de cette stratégie n'est pas, à mon sens, une lutte vicieuse telle que la présente Vilain, mais plutôt une sorte de dernier recours, voué bien évidemment à l'échec, pour tenter de retenir l'amant auprès de soi. Des fils du texte l'amant ne peut pas fuir, il ne peut plus lui échapper, il ne peut plus s'absenter. Aux absences de plus en plus fréquentes de l'amant s'oppose sa sur-présence textuelle, et l'amant évacue toute autre considération hors du journal. Ainsi, le journal permet à la diariste de s'assurer la visite du double de son amant, celui de papier, dont elle sait au moins qu'il lui sera fidèle et qu'il répondra à sa convocation. Dans cette optique, le journal intime permet de sauver la vie d'au moins deux façons: d'abord parce qu'il consigne l'existence et la réchappe de l'oubli, mais aussi puisqu'il permet de survivre aux absences désinvoltes de l'amant oublieux.

Je retiendrais donc l'importance du diaristique dans l'esthétique d'Annie Ernaux, en ce que l'auteure lie explicitement journal intime, témoignage et vérité. Cela est démontré, d'une part, par la contamination diaristique opérée dans les œuvres qui répondent à d'autres appellations génériques, et d'autre part, par la nécessité de compléter certains récits par la publication seconde du journal intime qui leur est attaché. Le journal se présente ici comme un supplément ${ }^{2}$ nécessaire, dont la fonction est finalement triple: consigner la vie alors même qu'elle se vit; permettre de survivre au trauma alors même qu'il est vécu; et témoigner du réel publiquement lorsqu'enfin il devient livre.

\footnotetext{
2 Je fais ici référence à la notion de texte supplément telle qu'elle a été élaborée par Jacques Derrida dans De la grammatologie, un texte second à la fois nécessaire et dangereux, parasitaire mais essentiel.
} 


\section{Bibliographie}

Derrida, Jacques. De la grammatologie. Paris, Minuit, coll. « Critique », 1967.

Didier, Béatrice. Le journal intime. Paris, PUF, 1976.

Ernaux, Annie. « Je ne suis pas sortie de ma nuit ». Paris, Gallimard, 1997.

Ernaux, Annie. Journal du dehors. Paris, Gallimard, 1993.

Ernaux, Annie. La vie extérieure. Paris, Gallimard, 2000.

Ernaux, Annie. Les armoires vides. Paris, Gallimard, 1974.

Ernaux, Annie. L'événement. Paris, Gallimard, 2000.

Ernaux, Annie. Passion simple. Paris, Gallimard,1991.

Ernaux, Annie. Se perdre. Paris, Gallimard, 2001.

Ernaux, Annie. Une femme. Paris, Gallimard, 1987.

Gasparini, Philippe. «Annie Ernaux, de Se perdre à Passion simple », dans Jean-Louis Jeannelle et Catherine Viollet (dir.), Genèse et autofiction, Louvain-la-Neuve, Bruylant-Academia, coll. « Au cœur des textes », 2007, 149-173.

Gasparini, Philippe. Est-il je ? Roman autobiographique et autofiction. Paris, Seuil, 2004.

Genette, Gérard. Figures III. Paris, Seuil, coll. « Poétique », 1972.

Girard, Alain. Le journal intime. Paris, PUF, 1963.

Havercroft, Barbara. «Auto/biographies croisées : L'étreinte d'Annie E. et Philippe V. », dans Robert Dion, Frances Fortier, Barbara Havercroft et Hans-Jürgen Lüsebrink (dir.), Vies en récit : Formes littéraires et médiatiques de la biographie et de l'autobiographie, Québec, Nota bene, coll. « Convergences », 2007, 159-186.

Leleu, Michèle. Les journaux intimes. Paris, PUF,1952.

Vilain, Philippe. Défense de Narcisse. Paris, Grasset, 2004.

http://www.brocku.ca/cfra/voixplurielles06-01/index.html 